\title{
AN ASYMPTOTIC SOLUTION TO A TWO-DIMENSIONAL EXIT PROBLEM ARISING IN POPULATION DYNAMICS*
}

\author{
H. ROOZENं
}

\begin{abstract}
A study is made of a two-dimensional stochastic system with small stochastic fluctuations arising in population biology. At the boundary of the state space the diffusion matrix becomes singular. By an asymptotic analysis, expressions are derived that determine the probability of exit at each of the two boundaries and the expectation and variance of the exit time. These expressions contain constants that can be computed numerically.
\end{abstract}

Key words. two-dimensional exit problem, diffusion matrix singular at boundary

AMS(MOS) subject classifications. 35C20, 35J25, 60J60, 60J70, 92A15

1. Introduction. Consider a two-dimensional stochastic system that has a stable deterministic equilibrium and in which the stochastic fluctuations are small. Various systems of this type have been studied in literature (see Matkowsky and Schuss [9], [10], Matkowsky, Schuss, and Tier [11], Hanson and Tier [7], Wazwaz and Hanson [14], [15]). With respect to the behaviour of the deterministic system at the boundary of the region under consideration, different cases can be distinguished: the deterministic vector field enters the region [9], or it is tangent to the boundary of the region. In the latter case, there may be no deterministic critical points on the boundary [10], or there may be [11]. It is assumed in [9], [10], and [11] that the diffusion tensor is nonsingular. The asymptotic theories for small stochastic fluctuations lead to expressions for the exit distribution and the (lowest) statistical moments of the exit time. An asymptotic analysis of a one-dimensional stochastic system in which the diffusion coefficient becomes singular at the boundary is given in [7], [14], and [15]. In this system, both the drift and the diffusion coefficients vanish linearly with the distance to the boundary.

The two-dimensional stochastic system treated in this paper arises in population dynamics [8], [12]. The diffusion matrix is diagonal and becomes singular at the boundary. There, the normal components of both the drift and the diffusion vanish linearly with the distance to the boundary. This system differs from the system treated in [11] in that the diffusion tensor becomes singular at the boundary, and from the system in [7], [14], and [15] in the dimension. Extending the methods presented in [7] and [11], asymptotic expressions are derived for the probabilities of exit at the two boundaries as well as the expectation and variance of the exit time.

Section 2 describes the stochastic model and formulates the boundary value problems with respect to exit boundary and exit time. In $\$ 3$ we find asymptotic expressions for the probability of exit at each of the boundaries, valid uniformly outside an asymptotic small neighbourhood of the origin. In $\S 4$, a derivation largely analogous to that in $\S 3$ leads to asymptotic expressions for the expectation and variance of the exit time that are uniformly valid. Section 5 is concerned with the numerical determination of constants that appear in the formulas obtained in $\S \S 3$ and 4 . As an example, $\S 6$ treats a predator-prey system.

2. The stochastic model and boundary value problems. We consider the twodimensional stochastic system with small stochastic fluctuations described by the

\footnotetext{
* Received by the editors November 30, 1987; accepted for publication (in revised form) October 27, 1988.

$\uparrow$ Centre for Mathematics and Computer Science, 1009 AB Amsterdam, the Netherlands.
} 
following Fokker-Planck (or forward Kolmogorov) equation:

$$
\frac{\partial v(x, t)}{\partial t}=M_{\varepsilon} v \equiv \sum_{i=1}^{2}\left[-\frac{\partial}{\partial x_{i}}\left[b_{i}(x) v(x, t)\right]+\frac{\varepsilon}{2} \frac{\partial^{2}}{\partial x_{i}^{2}}\left[a_{i}(x) v(x, t)\right]\right]
$$

in which $v$ is the probability density function and $0<\varepsilon \ll 1$ is a small parameter. The variables $x_{1}$ and $x_{2}$ denote the densities of two biological populations. The state space consists of the region $\bar{R}$ :

$$
R=\left\{\left(x_{1}, x_{2}\right) \mid x_{1}, x_{2} \text { real and }>0\right\} .
$$

The diffusion matrix is diagonal with elements:

$$
a_{1}(x)=x_{1}\left(a_{10}+a_{11} x_{1}+a_{12} x_{2}\right), \quad a_{2}(x)=x_{2}\left(a_{20}+a_{21} x_{1}+a_{22} x_{2}\right),
$$

in which the $a_{i j}$ are positive real numbers. This diffusion matrix is singular at $x_{1}=0$ and $x_{2}=0$. The drift vector is of the generalized Lotka-Volterra form:

$$
b_{1}(x)=x_{1}\left(b_{10}+b_{11} x_{1}+b_{12} x_{2}\right), \quad b_{2}(x)=x_{2}\left(b_{20}+b_{21} x_{1}+b_{22} x_{2}\right),
$$

where the $b_{i j}$ are real numbers that are restricted by assumptions made below. Thus, $x_{1}=0$ and $x_{2}=0$ are characteristic boundaries. The deterministic system

$$
\frac{d x_{1}}{d t}=b_{1}(x), \quad \frac{d x_{2}}{d t}=b_{2}(x)
$$

associated with the stochastic system (2.1) has the equilibria:

$$
\begin{aligned}
& (0,0), \\
& \left(0,-b_{20} / b_{22}\right), \\
& \left(-b_{10} / b_{11}, 0\right), \\
& x^{e}=\left(x_{1}^{e}, x_{2}^{e}\right) \equiv\left(\frac{b_{22} b_{10}-b_{12} b_{20}}{b_{21} b_{12}-b_{11} b_{22}}, \frac{b_{11} b_{20}-b_{21} b_{10}}{b_{21} b_{12}-b_{11} b_{22}}\right) .
\end{aligned}
$$

By assumption the critical points $(2.6 \mathrm{~b}),(2.6 \mathrm{c})$ lie on the positive $x_{2}$-axis, $x_{1}$-axis, respectively, with order $O(1)$ distance from the origin:

$$
-b_{20} / b_{22}>0, \quad-b_{20} / b_{22}=O(1), \quad-b_{10} / b_{11}>0, \quad-b_{10} / b_{11}=O(1),
$$

and are attracting along the $x_{2}$-axis and $x_{1}$-axis, respectively:

$$
b_{20}>0, \quad b_{10}>0 .
$$

The deterministic system has an equilibrium in $R$ with coordinates of order $O(1)$ :

$$
x_{1}^{e}>0, \quad x_{1}^{e}=O(1), \quad x_{2}^{e}>0, \quad x_{2}^{e}=O(1) .
$$

The following assumption is made with respect to the stability of the deterministic system at $x^{e}$. In the neighbourhood of $x^{e}$ we have by linearization of the deterministic vector field:

$$
b \equiv\left(b_{1}(x), b_{2}(x)\right)^{t} \approx B\left(x-x^{e}\right)
$$

where the matrix $B$ is given by

$$
B=\left(B_{i j}\right) \equiv\left(\frac{\partial b_{i}}{\partial x_{j}}\left(x^{e}\right)\right)=\left(b_{i j} x_{i}^{e}\right) .
$$

The eigenvalues of $B$ are

$$
\lambda_{1,2}=\frac{1}{2}\left[b_{11} x_{1}^{e}+b_{22} x_{2}^{e} \pm \sqrt{\left(b_{11} x_{1}^{e}+b_{22} x_{2}^{e}\right)^{2}-4\left(b_{11} b_{22}-b_{12} b_{21}\right) x_{1}^{e} x_{2}^{e}}\right] .
$$


The condition for stability of the deterministic system at $x^{e}$ is that the real parts of $\lambda_{1}$ and $\lambda_{2}$ are negative. With the use of the assumptions (A1)-(A3) this condition results in

$$
b_{11} b_{22}>b_{12} b_{21} \text {. }
$$

By the assumptions (A1)-(A4) the equilibria (2.6b), (2.6c) are saddle points. The equilibrium (2.6a) is an unstable node.

At the boundary $x_{i}=0$ we have

$$
\begin{aligned}
& J_{i}(x, t) \equiv b_{i}(x) v(x, t)-\frac{\varepsilon}{2} \frac{\partial}{\partial x_{i}}\left[a_{i}(x) v(x, t)\right]<0, \\
& b_{i}(x)=0, \quad a_{i}(x)=0,
\end{aligned}
$$

for $i=1,2$. By (2.10a) the probability current $J_{i}$ at $x_{i}=0$ is negative, which indicates that the boundary $x_{i}=0$ can be reached from $R$. Once $x_{i}=0$ has been reached, by (2.10b) it cannot be left. Thus, $x_{1}=0$ and $x_{2}=0$ are exit boundaries.

Starting away from $x_{1}=0$ and $x_{2}=0$, the stochastic system described above will likely remain in the neighbourhood of the stable equilibrium $x^{e}$ of the deterministic system for a long time. With small probabilities large excursions from $x^{e}$ occur. In such an excursion the system may exit at $x_{1}=0$ or $x_{2}=0$. This will happen within a finite time with probability one.

The boundary value problems describing exit are commonly defined on a bounded region. However, for the asymptotic analysis held in this paper, the use of the unbounded region $R$ and the boundary $\partial R$ defined by

$$
\partial R \equiv \bar{R} \backslash R=\left\{\left(x_{1}, x_{2}\right) \mid x_{1} x_{2}=0 \text { and } x_{1}+x_{2} \geqq 0\right\}
$$

will not lead to any difficulty. This is confirmed by results for the analogous onedimensional exit model, which can be computed explicitly.

To determine the probabilities of exit at $x_{1}=0$ and $x_{2}=0$, a study is made of the stationary backward Kolmogorov equation

$$
0=L_{F} u \equiv \sum_{i=1}^{2}\left[b_{i}(x) \frac{\partial u}{\partial x_{i}}+\frac{\varepsilon}{2} a_{i}(x) \frac{\partial^{2} u}{\partial x_{i}^{2}}\right] \text { in } R
$$

with the boundary condition

$$
u=f(x) \text { on } \partial R,
$$

in which

$$
u(x)=\int_{\partial R} f\left(x^{\prime}\right) P\left(x, x^{\prime}\right) d S_{x^{\prime}},
$$

where $P\left(x, x^{\prime}\right)$ is the probability of exit at $x^{\prime} \in \partial R$, starting from $x \in R$. With the definition

$$
f(x)= \begin{cases}1 & \text { for } x_{i}=0 \\ 0 & \text { else }\end{cases}
$$

the function $u(x)$ is the probability of exit at the particular boundary $x_{i}=0$, starting from $x \in R$. In this paper only boundary conditions of the form

$$
f(x)= \begin{cases}C_{b 1}, & x_{1}=0 \\ C_{b 2}, & x_{2}=0\end{cases}
$$

are considered with $C_{b 1}, C_{b 2}$ constants that are equal to either zero or one. 
Another point of interest is the determination of the expectation $\mathrm{E} T(x)$ and variance $\operatorname{Var} T(x)$ of the exit time $T(x)$, starting from $x \in R$. By

$$
\mathrm{E} T(x)=T_{1}, \quad \operatorname{Var} T(x)=T_{2}-T_{1}^{2},
$$

the expectation and variance of $T$ are expressed in the moments

$$
T_{i}(x)=\left\langle T^{i}\right\rangle
$$

of $T$, satisfying the equations

$$
L_{\varepsilon} T_{i}=g_{i}(x) \text { in } R
$$

and conditions

$$
T_{i}=0 \quad \text { on } \partial R
$$

for $i=1,2$ with

$$
g_{1}(x) \equiv-1, \quad g_{2}(x) \equiv-2 T_{1}(x) .
$$

Equation (2.17a) with $i=1$ is the Dynkin equation. Although higher moments can be determined as well, the analysis of the exit time in this paper is restricted to its expectation and variance. For a derivation of the boundary value problems (2.12), (2.17), the reader is referred to [4] and [13].

In biological terms, exit means extinction of a species. The expected exit time is a measure for the stochastic persistence of the ecosystem (see Ludwig [8]). The type of interaction between the two populations is mutualism for $b_{12}>0, b_{21}>0$, competition for $b_{12}<0, b_{21}<0$, and predation-prey in the other cases.

The motivation for the present study is the following one. In [12] we analyzed the exit problem for the system of populations described above by the method of Ludwig [8]. In that approach, the boundaries $x_{1}=0$ and $x_{2}=0$ of $R$ were replaced by the boundaries $x_{i}=l_{i}$, where the $l_{i}$ were small positive numbers, $i=1,2$. Thus the region $R$ was restricted to the smaller region $R_{l}$. Whereas the deterministic flow was tangent to the boundaries $x_{i}=0$ of $R$, it was directed inward to $R_{l}$ at the boundaries $x_{i}=l_{i}$ of $R_{l}$. Consequently, the asymptotics of Matkowsky and Schuss [9] could be applied to the problem of exit from $R_{l}$, as an approximation to the problem of exit from $R$. In the present paper we adopt a different point of view. The behaviour of the stochastic system near the boundaries $x_{1}=0$ and $x_{2}=0$ will be treated by a variant of [7] and [11]. In this approach we may take the limits $l_{i} \rightarrow 0$, where this was not allowed in the previous study [12]. Thus we obtain expressions to the problem of exit from $R$ without the need to approximate $R$ by a rather arbitrary smaller region $R_{l}$.

In [5] Gillespie treated an exit problem related to a multidimensional singular diffusion arising in genetics. His study differs in various aspects from the present one. In our model the deterministic system is more complicated since the behaviour near the boundaries $x_{1}=0$ and $x_{2}=0$ is dominated by the critical points $\left(0,-b_{20} / b_{22}\right)$ and $\left(-b_{10} / b_{11}, 0\right)$, where no such critical points are present in [5]. Moreover, for our model no explicit solution of the stationary forward equation is available and no zero probability flux condition holds. These facts give rise to a substantially more elaborate study.

3. The exit boundary. In this section the exit problem (2.12) with $f$ as in (2.14) is solved asymptotically for small $\varepsilon$. The solution contains an unknown constant. To obtain an expression for this constant we use an integral formula that results from the divergence theorem. In the integral formula, a formal solution of the forward equation adjoint to $(2.12 \mathrm{a})$ is needed. This adjoint equation is solved by the WKB-method (see 
Ludwig [8]). Near the boundaries $x_{1}=0$ and $x_{2}=0$, the solution of the adjoint equation is peaked at the critical points $\left(0,-b_{20} / b_{22}\right)$ and $\left(-b_{10} / b_{11}, 0\right)$, respectively. Neighbourhoods of these critical points play an important role in the subsequent analysis. This approach is a variant of the method of Matkowsky, Schuss, and Tier [11], that was indicated in Hanson and Tier [7].

3.1. The backward equation. An asymptotic analysis of the boundary value problem (2.12) reveals the existence of an outer solution, valid away from $x_{1}=0$ and $x_{2}=0$. Near these boundaries, an examination of different stretchings of the normal coordinate shows the presence of a boundary layer of width $O(\varepsilon)$. Inside the boundary layers, the diffusion parallel to the boundary is negligible, except near critical points of the deterministic system. Thus, the following regions are distinguished:

$$
\begin{array}{lll}
\text { region } A: & x_{1}=M_{1} \varepsilon, & x_{2}+b_{20} / b_{22}>M_{2} \sqrt{\varepsilon}, \\
\text { region } B: & x_{1}=M_{3} \varepsilon, \quad\left|x_{2}+b_{20} / b_{22}\right|=M_{4} \sqrt{\varepsilon}, \\
\text { region } C: & x_{1}=M_{5} \varepsilon, \quad M_{6} \varepsilon<x_{2}<-b_{20} / b_{22}-M_{7} \sqrt{\varepsilon}, \\
\text { region } D: & x_{1}=M_{8} \varepsilon, \quad x_{2}=M_{9} \varepsilon, \\
\text { region } A^{\prime}: & x_{1}+b_{10} / b_{11}>M_{10} \sqrt{\varepsilon}, \quad x_{2}=M_{11} \varepsilon, \\
\text { region } B^{\prime}: & \left|x_{1}+b_{10} / b_{11}\right|=M_{12} \sqrt{\varepsilon}, \quad x_{2}=M_{13} \varepsilon, \\
\text { region } C^{\prime}: & M_{14} \varepsilon<x_{1}<-b_{10} / b_{11}-M_{15} \sqrt{\varepsilon}, \quad x_{2}=M_{16} \varepsilon
\end{array}
$$

where the $M_{i}$ are arbitrary positive numbers independent of $\varepsilon$ (see Fig. 1).

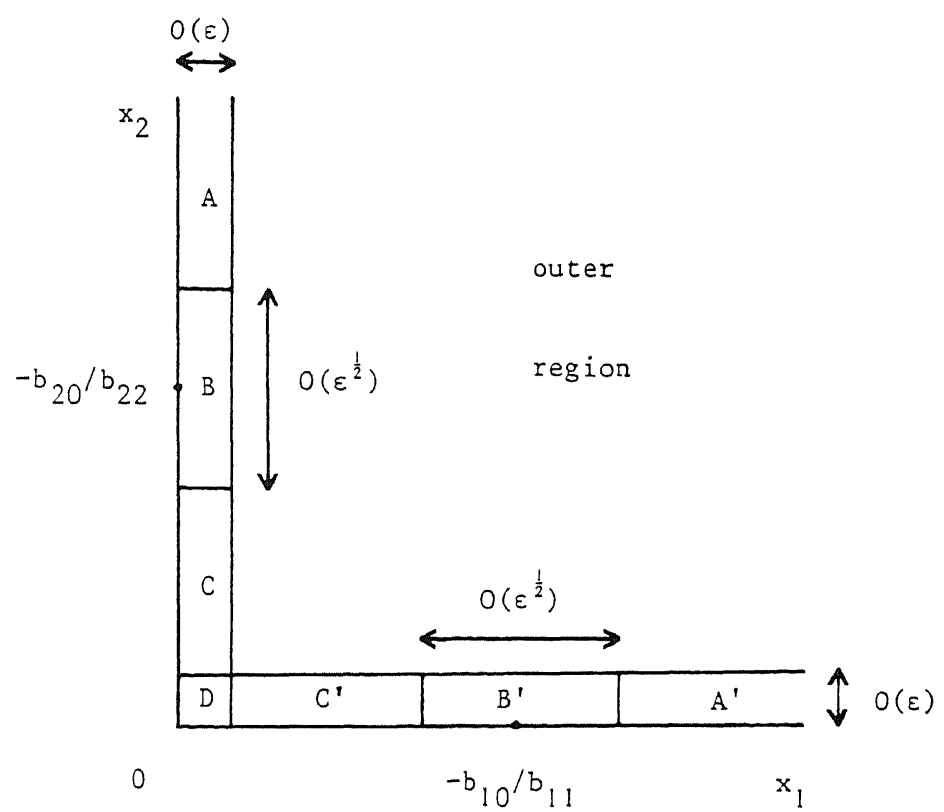

FIG. 1. The outer region and the boundary layer regions. In the regions $A, A^{\prime}, C, C^{\prime}$ the diffusion parallel to the boundary is negligible while this is not the case in the regions $B, B^{\prime}$, and $D$. 
3.1.1. The outer solution. The reduced equation corresponding to $(2.12 \mathrm{a})$ reads

$$
\sum_{i=1}^{2} b_{i}(x) \frac{\partial u}{\partial x_{i}}=0
$$

which has the solution

$$
u=C_{b},
$$

with $C_{b}$ a constant with respect to $x$, which is yet undetermined. An expression for $C_{b}$ will be found in $\S 3.3$. The solution (3.3) is valid in $R$ except near $x_{1}=0$ and $x_{2}=0$ because the boundary condition $(2.12 b),(2.14)$ cannot be satisfied.

3.1.2. The boundary layer solution in the regions $B$ and $\boldsymbol{B}^{\prime}$. Near the critical point $\left(0,-b_{20} / b_{22}\right)$ of the deterministic system, we introduce the stretched coordinates

$$
\bar{x}_{1}=x_{1} / \varepsilon, \quad \bar{x}_{2}=\left(x_{2}+b_{20} / b_{22}\right) / \sqrt{\varepsilon},
$$

and the boundary layer function

$$
U\left(\bar{x}_{1}, \bar{x}_{2}\right)=u\left(\varepsilon \bar{x}_{1},-b_{20} / b_{22}+\sqrt{\varepsilon} \bar{x}_{2}\right) .
$$

Substitution into $(2.12 \mathrm{a})$ leads to the boundary layer equation

$$
k_{1} \bar{x}_{1} \frac{\partial U}{\partial \bar{x}_{1}}+k_{2} \bar{x}_{1} \frac{\partial^{2} U}{\partial \bar{x}_{1}^{2}}-k_{3} \bar{x}_{2} \frac{\partial U}{\partial \bar{x}_{2}}+k_{4} \frac{\partial^{2} U}{\partial \bar{x}_{2}^{2}}=0
$$

in which

$$
\begin{aligned}
& k_{1}=b_{10}-b_{12} b_{20} / b_{22}, \quad k_{2}=\frac{1}{2}\left(a_{10}-a_{12} b_{20} / b_{22}\right), \\
& k_{3}=b_{20}, \quad k_{4}=-\frac{1}{2}\left(a_{20}-a_{22} b_{20} / b_{22}\right) b_{20} / b_{22} .
\end{aligned}
$$

From the assumptions (A1)-(A4) and the positivity of the $a_{i j}$ it follows that the constants $k_{i}$ are positive. By the separation of variables

$$
U\left(\bar{x}_{1}, \bar{x}_{2}\right)=w\left(\bar{x}_{1}\right) z\left(\bar{x}_{2}\right),
$$

(3.6) leads to the ordinary differential equations

$$
\begin{aligned}
& k_{2} \bar{x}_{1} \frac{d^{2} w}{d \bar{x}_{1}^{2}}+k_{1} \bar{x}_{1} \frac{d w}{d \bar{x}_{1}}-\lambda w=0, \\
& k_{4} \frac{d^{2} z}{d \bar{x}_{2}^{2}}-k_{3} \bar{x}_{2} \frac{d z}{d \bar{x}_{2}}+\lambda z=0,
\end{aligned}
$$

in which $\lambda$ is a separation constant. The general solution of (3.9a) is

$$
w\left(\bar{x}_{1}\right)=\left\{c_{1} W_{\lambda_{1}, 1 / 2}\left(-\tilde{x}_{1}\right)+c_{2} W_{-\lambda_{1}, 1 / 2}\left(\tilde{x}_{1}\right)\right\} \exp \left[-\tilde{x}_{1} / 2\right],
$$

in which

$$
\tilde{x}_{1}=k_{1} \bar{x}_{1} / k_{2}, \quad \lambda_{1}=\lambda / k_{1},
$$

$W_{\lambda_{1}, 1 / 2}$ and $W_{-\lambda_{1}, 1 / 2}$ are Whittaker functions [6], and $c_{1}, c_{2}$ are arbitrary constants. The general solution of $(3.9 \mathrm{~b})$ is

$$
z\left(\bar{x}_{2}\right)=\left\{c_{3} D_{\lambda_{2}}\left(\tilde{x}_{2}\right)+c_{4} D_{-\lambda_{2}-1}\left(i \tilde{x}_{2}\right)\right\} \exp \left[\tilde{x}_{2}^{2} / 4\right]
$$

in which

$$
\tilde{x}_{2}=\sqrt{k_{3} / k_{4}} \bar{x}_{2}, \quad \lambda_{2}=\lambda / k_{3},
$$


$D_{\lambda_{2}}$ and $D_{-\lambda_{2}-1}$ are parabolic cylinder functions [3], and $c_{3}, c_{4}$ are arbitrary constants. At $\bar{x}_{1}=0$ we have the boundary condition

$$
U\left(0, \bar{x}_{2}\right)=C_{b 1} .
$$

This condition can be satisfied only if $\lambda=0$. The matching condition with the outer solution (3.3) is stated as

$$
\lim _{\bar{x}_{1} \rightarrow \infty} U\left(\bar{x}_{1}, \bar{x}_{2}\right)=C_{b} .
$$

The boundary layer solution satisfying both conditions (3.14) and (3.15) is given by

$$
U\left(\bar{x}_{1}, \bar{x}_{2}\right)=C_{b}+\left(C_{b 1}-C_{b}\right) \exp \left[-\tilde{x}_{1}\right]
$$

or, in the original notation

$$
u\left(x_{1}, x_{2}\right)=C_{b}+\left(C_{b 1}-C_{b}\right) \exp \left[-k_{1} x_{1} / k_{2} \varepsilon\right] .
$$

The boundary layer region $B^{\prime}$ around the critical point $\left(-b_{10} / b_{11}, 0\right)$ yields a similar result:

$$
u\left(x_{1}, x_{2}\right)=C_{b}+\left(C_{b 2}-C_{b}\right) \exp \left[-k_{1}^{\prime} x_{2} / k_{2}^{\prime} \varepsilon\right],
$$

in which

$$
k_{1}^{\prime}=b_{20}-b_{21} b_{10} / b_{11}, \quad k_{2}^{\prime}=\frac{1}{2}\left(a_{20}-a_{21} b_{10} / b_{11}\right) .
$$

3.1.3. The boundary layer solution in the regions $A, A^{\prime}$ and $C, C^{\prime}$. Introduction of the stretched coordinate $\bar{x}_{1}$ in (3.4) and the boundary layer function

$$
U\left(\bar{x}_{1}, x_{2}\right)=u\left(\varepsilon \bar{x}_{1}, x_{2}\right)
$$

into equation (2.12a) leads to the boundary layer equation

$$
\bar{x}_{1}\left(b_{10}+b_{12} x_{2}\right) \frac{\partial U}{\partial \bar{x}_{1}}+x_{2}\left(b_{20}+b_{22} x_{2}\right) \frac{\partial U}{\partial x_{2}}+\bar{x}_{1} \frac{1}{2}\left(a_{10}+a_{12} x_{2}\right) \frac{\partial^{2} U}{\partial \bar{x}_{1}^{2}}=0 .
$$

To make this equation separable, the variable $\bar{x}_{1}$ is replaced by the new variable

$$
y=\bar{x}_{1} \gamma\left(x_{2}\right),
$$

with the function $\gamma$ still to be determined. Equation (3.20) becomes

$$
y \frac{\partial^{2} W}{\partial y^{2}}+\Gamma\left(x_{2}\right) y \frac{\partial W}{\partial y}+\frac{x_{2}\left(b_{20}+b_{22} x_{2}\right)}{\frac{1}{2}\left(a_{10}+a_{12} x_{2}\right) \gamma} \frac{\partial W}{\partial x_{2}}=0,
$$

where

$$
W\left(y, x_{2}\right)=U\left(\bar{x}_{1}, x_{2}\right),
$$

and

$$
\Gamma\left(x_{2}\right) \equiv \frac{1}{\frac{1}{2}\left(a_{10}+a_{12} x_{2}\right) \gamma}\left[b_{10}+b_{12} x_{2}+x_{2}\left(b_{20}+b_{22} x_{2}\right) \gamma^{\prime} / \gamma\right] .
$$

The function $\gamma$ is chosen such that

$$
\Gamma\left(x_{2}\right)=1 .
$$

Then (3.24) is a Bernoulli equation. In terms of the reciprocal $\gamma^{-1}$, it is a linear equation that can be solved by the method of variation of constants [1]. Since (3.24), (3.25) is a first-order differential equation, there is one integration constant. This constant 
follows from a matching condition (see below). The partial differential equation (3.22) with (3.25) can be solved by separation of variables:

$$
W\left(y, x_{2}\right)=w(y) z\left(x_{2}\right)
$$

which leads to the ordinary differential equations

$$
\begin{aligned}
& y \frac{d^{2} w}{d y^{2}}+y \frac{d w}{d y}-\lambda w=0, \\
& \frac{x_{2}\left(b_{20}+b_{22} x_{2}\right)}{\frac{1}{2}\left(a_{10}+a_{12} x_{2}\right) \gamma} \frac{d z}{d x_{2}}+\lambda z=0,
\end{aligned}
$$

in which $\lambda$ is a separation constant. To satisfy the matching conditions

$$
U\left(0, x_{2}\right)=C_{b 1}, \quad \lim _{\bar{x}_{1} \rightarrow \infty} U\left(\bar{x}_{1}, x_{2}\right)=C_{b},
$$

$\lambda$ must equal zero and the solution of (3.20), (3.28) is obtained as

$$
U\left(\bar{x}_{1}, x_{2}\right)=C_{b}+\left(C_{b 1}-C_{b}\right) \exp \left[-\gamma\left(x_{2}\right) \bar{x}_{1}\right]
$$

or, in the original notation

$$
u\left(x_{1}, x_{2}\right)=C_{b}+\left(C_{b 1}-C_{b}\right) \exp \left[-\gamma\left(x_{2}\right) x_{1} / \varepsilon\right] .
$$

The integration constant in the problem (3.24), (3.25) for $\gamma$ is chosen such that (3.29) matches the solution (3.16), that is, by the condition

$$
\lim _{x_{2} \rightarrow-b_{20} / b_{22}} \gamma\left(x_{2}\right)=\frac{k_{1}}{k_{2}} \text {. }
$$

For future purposes we remark that at $x_{2}=-b_{20} / b_{22}$ and $x_{2}=0$ the function $\gamma^{-1}$ has the Taylor series expansions:

$$
\begin{aligned}
& \gamma^{-1}\left(x_{2}\right)=\frac{k_{2}}{k_{1}}+\frac{a_{12} b_{10}-a_{10} b_{12}}{2 k_{1}\left(b_{20}+k_{1}\right)}\left(x_{2}+\frac{b_{20}}{b_{22}}\right)+\cdots, \\
& \gamma^{-1}\left(x_{2}\right)=\frac{a_{10}}{2 b_{10}}-\frac{a_{12} b_{10}-a_{10} b_{12}}{2 b_{10}\left(b_{20}-b_{10}\right)} x_{2}+\cdots,
\end{aligned}
$$

respectively.

The boundary layer regions $A^{\prime}$ and $C^{\prime}$ along the $x_{1}$-axis are treated similarly. There the solution is given by

$$
u\left(x_{1}, x_{2}\right)=C_{b}+\left(C_{b 2}-C_{b}\right) \exp \left[-\tilde{\gamma}\left(x_{1}\right) x_{2} / \varepsilon\right]
$$

in which $\tilde{\gamma}\left(x_{1}\right)$ solves a Bernoulli problem analogous to (3.24), (3.25), (3.30). The treatment above in the direction along the boundary leads to a correct result only for constant boundary conditions. Readers interested in boundary conditions (2.12b) with nonconstant $f$ are referred to the approach in [11].

3.1.4. Summary. A boundary layer analysis in the region $D$ leads to a complicated expression for $u$. This is due in part to the conditions to be satisfied by $u$. Besides matching conditions with the solutions in $C$ (see $(3.29 \mathrm{~b})$ and $(3.31 \mathrm{~b})$ with $\left.x_{2}=0\right)$ and $C^{\prime}$ these are the conditions $(2.12 \mathrm{~b}),(2.14)$ along $x_{1}=0$ and $x_{2}=0$. To retain simplicity, and since it will turn out in the remainder of $\S 3$ that for small $\varepsilon$ the asymptotic small region $D$ is not important to the dynamics elsewhere, we discard the solution in this region. It is easily verified that the results of $\S 3.1 \mathrm{can}$ then be summarized as follows. 
The uniform asymptotic expansion for small $\varepsilon$ in $\bar{R} \backslash \bar{D}, D$ an $O(\varepsilon)$-neighbourhood of the origin, of the boundary value problem $(2.12),(2.14)$ is given by

$$
\begin{aligned}
u(x)= & \frac{1}{C_{b}}\left\{C_{b}+\left(C_{b 1}-C_{b}\right) \exp \left[-\gamma\left(x_{2}\right) x_{1} / \varepsilon\right]\right\} \\
& \times\left\{C_{b}+\left(C_{b 2}-C_{b}\right) \exp \left[-\tilde{\gamma}\left(x_{1}\right) x_{2} / \varepsilon\right]\right\},
\end{aligned}
$$

in which $\gamma, \tilde{\gamma}$ solve Bernoulli problems as discussed in $\S 3.1 .3$. Expression (3.33) is the uniform asymptotic expansion in $\bar{R}$ of the boundary value problem (2.12) with the boundary conditions

$$
\begin{aligned}
& u\left(0, x_{2}\right)=C_{b 1}\left\{1+\left(C_{b 2} / C_{b}-1\right) \exp \left[-2 b_{20} x_{2} / a_{20} \varepsilon\right]\right\}, \\
& u\left(x_{1}, 0\right)=C_{b 2}\left\{1+\left(C_{b 1} / C_{b}-1\right) \exp \left[-2 b_{10} x_{1} / a_{10} \varepsilon\right]\right\},
\end{aligned}
$$

which are different from the boundary conditions (2.14) in the region $D$. The remainder of $\S 3$ concerns the determination of $C_{b}$, which is yet unknown.

3.2. The adjoint equation. The forward equation adjoint to (2.12a) is given by

$$
M_{\varepsilon} v=0
$$

with the operator $M_{\varepsilon}$ defined in (2.1). The function $v(x)$ describes the probability density corresponding to the (quasi-) stationary state of the system (2.1). The solution of equation (3.35) is needed in $\S 3.3$.

3.2.1. The WKB-approximation. A solution of (3.35) is sought in the form of the WKB-Ansatz [8]:

$$
v\left(x_{1}, x_{2}\right)=w\left(x_{1}, x_{2}\right) \exp \left[-Q\left(x_{1}, x_{2}\right) / \varepsilon\right], \quad \varepsilon \rightarrow 0,
$$

where

$$
\begin{aligned}
& Q\left(x_{1}^{e}, x_{2}^{e}\right)=0, \\
& w\left(x_{1}^{e}, x_{2}^{e}\right)=1 \quad \text { (normalization). }
\end{aligned}
$$

Substitution of this form into (3.35) leads to leading order $O\left(\varepsilon^{-1}\right)$ to the eikonal equation:

$$
\sum_{i=1}^{2}\left[b_{i} \frac{\partial Q}{\partial x_{i}}+\frac{1}{2} a_{i}\left(\frac{\partial Q}{\partial x_{i}}\right)^{2}\right]=0
$$

and to order $O\left(\varepsilon^{0}\right)$ to the transport equation:

$$
\sum_{i=1}^{2}\left[\frac{\partial}{\partial x_{i}}\left(b_{i} w\right)+\frac{\partial Q}{\partial x_{i}} \frac{\partial}{\partial x_{i}}\left(a_{i} w\right)+\frac{1}{2} a_{i} w \frac{\partial^{2} Q}{\partial x_{i}^{2}}\right]=0 .
$$

The numerical computation of the functions $Q$ and $w$ subject to the conditions (3.36b), $(3.36 \mathrm{c})$ is treated in $\S 5$.

3.2.2. Behaviour near the boundary. To investigate the asymptotic behaviour of $Q$ in the $x_{2}$-direction for small $x_{1}$, the expansion

$$
Q\left(x_{1}, x_{2}\right)=\bar{Q}_{0}\left(x_{2}\right)+\bar{Q}_{1}\left(x_{2}\right) x_{1}+\frac{1}{2} \bar{Q}_{2}\left(x_{2}\right) x_{1}^{2}+\cdots
$$

is substituted into (3.37). Terms of order $O\left(x_{1}^{0}\right)$ are collected, which results in

$$
\frac{d \bar{Q}_{0}}{d x_{2}}=-\frac{b_{20}+b_{22} x_{2}}{\frac{1}{2}\left(a_{20}+a_{22} x_{2}\right)} \text {. }
$$


This expression indicates that inside the interval $x_{2} \in[0, \infty)$ the only extremum of $\bar{Q}_{0}$ is a minimum, situated at the critical point $x_{2}=-b_{20} / b_{22}$. By (3.36a) the probability density function $v$ is sharply peaked at this critical point. Therefore, the probability of meeting the stochastic system in the boundary layer $x_{1}=O(\varepsilon)$, asymptotically equals the probability of meeting the system in the boundary layer region $B$.

To study the WKB-solution in the region $B$ the new variable

$$
\hat{x}_{2}=x_{2}+b_{20} / b_{22}
$$

is introduced and $Q$ is approximated by the Taylor series expansion:

$$
Q\left(x_{1}, x_{2}\right)=Q_{0}+Q_{2} \hat{x}_{2}+Q_{1} x_{1}+\frac{1}{2} Q_{3} \hat{x}_{2}^{2}+\cdots .
$$

Note that $x_{1}$ is of the order $O\left(\hat{x}_{2}^{2}\right)$ in the region B. Substitution of (3.42) into the eikonal equation (3.37) determines the constants

$$
Q_{2}=0, \quad Q_{1}=-k_{1} / k_{2}, \quad Q_{3}=k_{3} / k_{4},
$$

and leaves the constant $Q_{0}$ undetermined. The value of $Q_{0}$ is obtained by solving the problem (3.35), (3.36) numerically.

A boundary layer analysis is carried out to reveal the behaviour of the transport function $w$ in the region $B$. The WKB-solution of the stationary forward equation of a one-dimensional variant of our model can be calculated explicitly and indicates a singular behaviour. The stretched coordinates (3.4) and the boundary layer function

$$
V\left(\bar{x}_{1}, \bar{x}_{2}\right)=v\left(\varepsilon \bar{x}_{1},-b_{20} / b_{22}+\sqrt{\varepsilon} \bar{x}_{2}\right)
$$

are introduced. Substitution into (3.35) leads to the boundary layer equation:

$$
-k_{1} \frac{\partial}{\partial \bar{x}_{1}}\left(\bar{x}_{1} V\right)+k_{2} \frac{\partial^{2}}{\partial \bar{x}_{1}^{2}}\left(\bar{x}_{1} V\right)+k_{3} \frac{\partial}{\partial \bar{x}_{2}}\left(\bar{x}_{2} V\right)+k_{4} \frac{\partial^{2} V}{\partial \bar{x}_{2}^{2}}=0,
$$

with the $k_{i}$ defined in (3.7). By the separation assumption

$$
V\left(\bar{x}_{1}, \bar{x}_{2}\right)=r\left(\bar{x}_{1}\right) s\left(\bar{x}_{2}\right)
$$

(3.45) leads to the ordinary differential equations

$$
\begin{aligned}
& k_{2} \frac{d^{2}}{d \bar{x}_{1}^{2}}\left(\bar{x}_{1} r\right)-k_{1} \frac{d}{d \bar{x}_{1}}\left(\bar{x}_{1} r\right)-\mu r=0, \\
& k_{4} \frac{d^{2} s}{d \bar{x}_{2}^{2}}+k_{3} \frac{d}{d \bar{x}_{2}}\left(\bar{x}_{2} s\right)+\mu s=0,
\end{aligned}
$$

in which $\mu$ is a separation constant. The general solution of (3.47a) is

$$
r\left(\bar{x}_{1}\right)=\left\{c_{1} W_{\mu_{1}, 1 / 2}\left(-\tilde{x}_{1}\right)+c_{2} W_{-\mu_{1}, 1 / 2}\left(\tilde{x}_{1}\right)\right\} \tilde{x}_{1}^{-1} \exp \left[\tilde{x}_{1} / 2\right],
$$

with

$$
\mu_{1}=\mu / k_{1}
$$

$c_{1}, c_{2}$ arbitrary constants, and $\tilde{x}_{1}$ defined in (3.11). The general solution of $(3.47 \mathrm{~b})$ is

$$
s\left(\bar{x}_{2}\right)=\left\{c_{3} D_{\mu_{2}}\left(\tilde{x}_{2}\right)+c_{4} D_{-\mu_{2}-1}\left(i \tilde{x}_{2}\right)\right\} \exp \left[-\tilde{x}_{2}^{2} / 4\right] \text {, }
$$

$$
\mu_{2}=\mu / k_{3},
$$

$c_{3}, c_{4}$ arbitrary constants and $\tilde{x}_{2}$ defined in (3.13). Putting

$$
\mu=0, \quad c_{2}=0, \quad c_{4}=0
$$


the boundary layer solution

$$
v\left(\bar{x}_{1}, \bar{x}_{2}\right)=\text { const. } \tilde{x}_{1}^{-1} \exp \left[\tilde{x}_{1}-\tilde{x}_{2}^{2} / 2\right]
$$

is obtained. The leading order part of the WKB-solution (3.36a) with $Q$ given by (3.42), (3.43) agrees with the exponential function in the boundary layer solution (3.53). The solution (3.53) indicates that the transport equation $w$ behaves according to

$$
w \sim x_{1}^{-1}
$$

in the region $B$. Substitution of the expansion

$$
w\left(x_{1}, x_{2}\right)=x_{1}^{-1}\left(w_{0}+w_{2} \hat{x}_{2}+w_{1} x_{1}+\frac{1}{2} w_{3} \hat{x}_{2}^{2}+\cdots\right)
$$

into the transport equation (3.38) and using (3.42), (3.43) leaves the constant $w_{0}$ undetermined. Its value is obtained by solving the problem (3.35), (3.36) numerically.

As a conclusion, in the boundary layer region $B$ the WKB-solution (3.36) behaves as

$$
v\left(x_{1}, x_{2}\right)=C_{1}(\varepsilon) x_{1}^{-1} \exp \left[\left(k_{1} x_{1} / k_{2}-k_{3} \hat{x}_{2}^{2} / 2 k_{4}\right) / \varepsilon\right]
$$

in which

$$
C_{1}(\varepsilon)=w_{0} \exp \left[-Q_{0} / \varepsilon\right]
$$

where $w_{0}, Q_{0}$ as in (3.55), (3.42), respectively, have to be determined numerically. A similar result can be derived in the boundary layer region $B^{\prime}$. There, the WKB-solution is

$$
v\left(x_{1}, x_{2}\right)=C_{2}(\varepsilon) x_{2}^{-1} \exp \left[\left(k_{1}^{\prime} x_{2} / k_{2}^{\prime}-k_{3}^{\prime} \hat{x}_{1}^{2} / 2 k_{4}^{\prime}\right) / \varepsilon\right]
$$

where the constants in $C_{2}$, which is the analogue of $C_{1}$, have to be determined numerically. The constants $k_{3}^{\prime}, k_{4}^{\prime}$ are given by

$$
k_{3}^{\prime}=b_{10}, \quad k_{4}^{\prime}=-\frac{1}{2}\left(a_{10}-a_{11} b_{10} / b_{11}\right) b_{10} / b_{11},
$$

and $\hat{x}_{1}$ by

$$
\hat{x}_{1}=x_{1}+b_{10} / b_{11} \text {. }
$$

3.3. Application of the divergence theorem. Using the divergence theorem the following integral relation can be derived:

$$
\int_{R^{\prime}}\left(v L_{\varepsilon} u-u M_{\varepsilon} v\right) d R^{\prime}=\int_{\partial R^{\prime}} \sum_{i=1}^{2} \nu_{i}\left[\frac{\varepsilon}{2} a_{i}\left(v \frac{\partial u}{\partial x_{i}}-u \frac{\partial v}{\partial x_{i}}\right)+\left(b_{i}-\frac{\varepsilon}{2} \frac{\partial a_{i}}{\partial x_{i}}\right) u v\right] d S
$$

where $R^{\prime}$ is a region with boundary $\partial R^{\prime}$ on which the operators $L_{\varepsilon}, M_{\varepsilon}$ are defined and $\nu$ denotes the outward normal on $\partial R^{\prime}$. In the right side of (3.61) $v$ and its conormal derivative must be evaluated at the boundary. By (3.56), (3.58) these functions become singular at $x_{1}=0$ and $x_{2}=0$. To avoid singular functions, $R^{\prime}$ is chosen as a slight modification of the region $R$ :

$$
R^{\prime}=\left\{\left(x_{1}, x_{2}\right) \mid x_{1}, x_{2}>\delta\right\}
$$

and

$$
\partial R^{\prime} \equiv \bar{R}^{\prime} \backslash R^{\prime}=\left\{\left(x_{1}, x_{2}\right) \mid\left(x_{1}-\delta\right)\left(x_{2}-\delta\right)=0 \text { and } x_{1}+x_{2} \geqq 2 \delta\right\},
$$

with

$$
0<\delta \ll \varepsilon
$$


By (2.12a) and (3.35) the left side of (3.61) equals zero. First the boundary $x_{1}=\delta$ of $R^{\prime}$ is considered. There, the right side of (3.61) is written as

$$
\int_{\delta}^{\infty}\left[\frac{\varepsilon}{2} a_{1}\left(-v \frac{\partial u}{\partial x_{1}}+u \frac{\partial v}{\partial x_{1}}\right)-\left(b_{1}-\frac{\varepsilon}{2} \frac{\partial a_{1}}{\partial x_{1}}\right) u v\right]_{x_{1} \ldots \delta} d x_{2} .
$$

The only significant contribution to this integral comes from the boundary layer region $B$. Using the expression (3.16b) for $u$ and (3.56) for $v$, the integrand in (3.65) is evaluated. Subsequently the limit $\delta \rightarrow 0$ is taken, and asymptotically for small $\varepsilon$ the following result is obtained:

$$
\begin{aligned}
\int_{0}^{\infty}\left[\left(a_{10}-b_{10} k_{2} / k_{1}\right) C_{b 1}-\frac{1}{2} a_{10} C_{b}+\left\{\left(a_{12}-b_{12} k_{2} / k_{1}\right) C_{b 1}-\frac{1}{2} a_{12} C_{b}\right\} x_{2}\right] \\
\cdot\left(\frac{k_{1}}{k_{2}}\right) C_{1}(\varepsilon) \exp \left[-k_{3} \hat{x}_{2}^{2} / 2 k_{4} \varepsilon\right] d x_{2} .
\end{aligned}
$$

This integral is evaluated by the method of Laplace [1]. The boundary $x_{2}=\delta$ is treated similarly. Both results are used in the divergence formula (3.61) to obtain the following expression for $C_{b}$ :

$$
C_{b}=\frac{C_{b_{1}} C_{1}(\varepsilon) K_{1}+C_{b_{2}} C_{2}(\varepsilon) K_{2}}{C_{1}(\varepsilon) K_{1}+C_{2}(\varepsilon) K_{2}}
$$

with the abbreviations

$$
K_{1}=k_{1} \sqrt{k_{4} / k_{3}}, \quad K_{2}=k_{1}^{\prime} \sqrt{k_{4}^{\prime} / k_{3}^{\prime}} .
$$

Expression (3.67) completes the analysis of $\S 3$. With (3.33) the following result is obtained. Denoting the probability of exit at the boundary $x_{\varepsilon}=0$, starting at $x$, by $u_{i}(x)$, we have

(3.69a) $u_{1}(x)=\frac{C_{1}(\varepsilon) K_{1}+C_{2}(\varepsilon) K_{2} \exp \left[-\gamma\left(x_{2}\right) x_{1} / \varepsilon\right]}{C_{1}(\varepsilon) K_{1}+C_{2}(\varepsilon) K_{2}}\left[1-\exp \left[-\tilde{\gamma}\left(x_{1}\right) x_{2} / \varepsilon\right]\right]$

(3.69b) $u_{2}(x)=\frac{C_{1}(\varepsilon) K_{1} \exp \left[-\tilde{\gamma}\left(x_{1}\right) x_{2} / \varepsilon\right]+C_{2}(\varepsilon) K_{2}}{C_{1}(\varepsilon) K_{1}+C_{2}(\varepsilon) K_{2}}\left[1-\exp \left[-\gamma\left(x_{2}\right) x_{1} / \varepsilon\right]\right]$

asymptotically for small $\varepsilon$ in $\bar{R} \backslash \bar{D}, D$ an $O(\varepsilon)$-neighbourhood of the origin. It is easily verified that in the region $\bar{R} \backslash \bar{D}$, the expressions (3.69a) and $(3.69 \mathrm{~b})$ add up to one. Rewriting (3.57) and the analogous expression for $C_{2}$ as

$$
C_{i}(\varepsilon)=w_{(i)} \exp \left[-Q_{(n)} / \varepsilon\right], \quad i=1,2,
$$

and using the fact that $w_{0 i}, K_{i}$ are order $O(1)$ constants, we can simplify (3.69). In the case $Q_{011}<Q_{012}$ we find

$$
\left.u_{1}(x) \sim 1-\exp \left[-\tilde{\gamma}\left(x_{1}\right) x_{2} / \varepsilon\right], \quad u_{2}(x) \sim \exp \mid-\tilde{\gamma}\left(x_{1}\right) x_{2} / \varepsilon\right],
$$

and in the case $Q_{01}>Q_{12}$

$$
u_{1}(x) \sim \exp \left[-\gamma\left(x_{2}\right) x_{1} / \varepsilon\right], \quad u_{2}(x) \sim 1-\exp \left[-\gamma\left(x_{2}\right) x_{1} / \varepsilon\right] .
$$

4. The expectation and variance of the exit time. In this section the boundary value problems (2.17) are solved asymptotically for small $\varepsilon$. Assume that $T_{i}(x)$ is of the form

$$
T_{i}(x)=C_{T, i}(\varepsilon) \tau_{i}(x),
$$

in which

$$
C_{T, i}^{1}(\varepsilon) g_{1}(x)=o(\varepsilon), \quad \varepsilon \rightarrow 0 .
$$


Substitution of (4.1) into (2.17a) yields to leading order the reduced equation

$$
\sum_{j=1}^{2} b_{j}(x) \frac{\partial \tau_{i}}{\partial x_{j}}=0
$$

which is solved by a constant that is taken to be one without loss of generality (any other value can be incorporated in $C_{T, i}$ ):

$$
\tau_{i}(x)=1 \text {. }
$$

This is the outer solution, valid away from $O(\varepsilon)$ boundary layers along $x_{1}=0$ and $x_{2}=0$. A boundary layer analysis can be held as in $\S 3$, with $u$ replaced by $\tau_{i}$. The only difference is in the boundary condition, for this case stated by $(2.17 \mathrm{~b})$. The following uniform asymptotic expansion for $\tau_{i}$ is obtained:

$$
\tau_{i}\left(x_{1}, x_{2}\right)=\left[1-\exp \left[-\gamma\left(x_{2}\right) x_{1} / \varepsilon\right]\right]\left[1-\exp \left[-\tilde{\gamma}\left(x_{1}\right) x_{2} / \varepsilon\right]\right],
$$

valid in $R$ (the region $D$ included, due to the boundary conditions, which are simpler here than in $\S 3$ ). The unknown $C_{T, i}(\varepsilon)$ are determined using the integral relation (3.61) with $u$ replaced by $T_{i}$. After some calculation, this integral relation reduces to

$$
-\frac{2}{\varepsilon} \int_{\delta}^{\infty} \int_{\delta}^{\infty} v g_{i} d x_{1} d x_{2}=\int_{\delta}^{\infty}\left[a_{1} v \frac{\partial T_{i}}{\partial x_{1}}\right]_{x_{1}=\delta} d x_{2}+\int_{\delta}^{\infty}\left[a_{2} v \frac{\partial T_{i}}{\partial x_{2}}\right]_{x_{2}=\delta} d x_{1}
$$

On the right side, the largest contributions to the integrals are from the boundary layer regions $B$ and $B^{\prime}$. These integrals are evaluated by the method of Laplace, using expressions (4.1), (4.5) with

$$
\gamma\left(x_{2}\right)=k_{1} / k_{2}, \quad \tilde{\gamma}\left(x_{1}\right)=k_{1}^{\prime} / k_{2}^{\prime}
$$

for $T$ and expressions (3.56), (3.58) for $v$. The left side of (4.6) is evaluated using the WKB-expression (3.36a) for $v$ and the method of Laplace for double integrals. Letting $\delta \rightarrow 0$, the following expressions are found for $C_{T, i}(\varepsilon)$ :

$$
C_{T, 1}(\varepsilon)=\frac{\sqrt{2 \pi \varepsilon / H e\left(x^{e}\right)}}{C_{1}(\varepsilon) K_{1}+C_{2}(\varepsilon) K_{2}}, \quad C_{T, 2}(\varepsilon)=2 C_{T, 1}^{2}(\varepsilon)
$$

in which $\mathrm{He}\left(x^{e}\right)$ is the determinant of the Hessian matrix of $Q$ at $x^{e}$. With $Q^{*}=$ $\min \left(Q_{01}, Q_{02}\right)$ (see (3.70)), and using the fact that $w_{0 i}, K_{i}, H e\left(x^{e}\right)$ are of order $O(1)$, $C_{T, 1}(\varepsilon)$ is of the order

$$
C_{T, 1}(\varepsilon) \sim \sqrt{\varepsilon} \exp \left[Q^{*} / \varepsilon\right] .
$$

In the evaluation of the left side of (4.6) we let $\varepsilon \rightarrow 0$, while the WKB-expression (3.36a) for $v$ fails to be integrable in this limit. This procedure was proposed by Ludwig [8]. Its correctness has not been proven. See also the remarks in [7] at this point. By (2.15) the resulting uniform asymptotic expansions in $\bar{R}$ of the expectation and variance of the exit time are given as

$$
\begin{aligned}
& \operatorname{ET}(x)=C_{T, 1}(\varepsilon)\left[1-e^{-\gamma\left(x_{2}\right) x_{1} / \varepsilon}\right]\left[1-e^{-\tilde{\gamma}\left(x_{1}\right) x_{2} / \varepsilon}\right] \\
& \operatorname{Var} T(x)=C_{T, 1}^{2}(\varepsilon)\left[1-\left\{\exp \left[-\gamma\left(x_{2}\right) x_{1} / \varepsilon\right]\right.\right. \\
& \left.\left.\quad+\exp \left[-\tilde{\gamma}\left(x_{1}\right) x_{2} / \varepsilon\right]-\exp \left[-\gamma\left(x_{2}\right) x_{1} / \varepsilon-\tilde{\gamma}\left(x_{1}\right) x_{2} / \varepsilon\right]\right\}^{2}\right],
\end{aligned}
$$

respectively.

5. Numerical determination of the WKB-solution. To obtain the constants $Q_{01}$, $w_{01}, Q_{02}, w_{02}$ in (3.70), the WKB-solution (3.36) of the adjoint equation (3.35) is 
determined numerically. By the Hamilton-Jacobi theory [2], the eikonal equation (3.37) is written in terms of the Hamiltonian $H$ :

$$
H(x, p) \equiv \sum_{i=1}^{2}\left[b_{i} p_{i}+\frac{1}{2} a_{i} p_{i}^{2}\right]=0
$$

where

$$
p_{i}=\frac{\partial Q}{\partial x_{i}} .
$$

The corresponding system of bicharacteristics reads

$$
\begin{aligned}
& \frac{d x_{i}}{d s}=\frac{\partial H}{\partial p_{i}}=b_{i}+a_{i} p_{i} \quad(i=1,2), \\
& \frac{d p_{i}}{d s}=-\frac{\partial H}{\partial x_{i}}=-\sum_{j=1}^{2}\left[\frac{\partial b_{j}}{\partial x_{i}} p_{j}+\frac{1}{2} \frac{\partial a_{j}}{\partial x_{i}} p_{j}^{2}\right] \quad(i=1,2)
\end{aligned}
$$

with $s$ a parameter along the characteristics. The rate of change of $Q$ with $s$ is given by

$$
\frac{d Q}{d s}=-H+\sum_{i=1}^{2} \frac{d x_{i}}{d s} p_{i}=\sum_{i=1}^{2} \frac{1}{2} a_{i} p_{i}^{2} \quad(\text { which is } \geqq 0) .
$$

At $s=0$ all characteristics start in a neighbourhood of the equilibrium

$$
x=x^{e}, \quad p=0, \quad Q=0,
$$

of the system (5.2). The initial position of a characteristic is specified on a circle around $x^{e}$ with radius $r \ll 1$, by the variable $\theta$ :

$$
x_{1}=x_{1}^{e}+r \cos \theta, \quad x_{2}=x_{2}^{e}+r \sin \theta .
$$

The corresponding initial values of $p_{1}, p_{2}, Q$ are obtained by the following local analysis. In the neighbourhood of (5.3), $Q$ is approximated by the quadratic form:

$$
Q \approx \frac{1}{2}\left(x-x^{e}\right)^{t} P\left(x-x^{e}\right),
$$

in which $P$ is a symmetric matrix and $t$ denotes the transpose. It follows that

$$
p=\frac{d Q}{d x} \approx P\left(x-x^{e}\right)
$$

Substitution of the approximations (5.6), (2.7) and the approximation

$$
\left(\begin{array}{cc}
a_{1}\left(x^{e}\right) & 0 \\
0 & a_{2}\left(x^{e}\right)
\end{array}\right)=\left(\begin{array}{cc}
A_{1} & 0 \\
0 & A_{2}
\end{array}\right) \equiv A
$$

of the diffusion matrix into the eikonal equation (3.37) leads to the matrix equation

$$
P A P+P B+B^{t} P=0,
$$

which is solved to give

$$
\begin{aligned}
P= & \frac{-2\left(B_{11}+B_{22}\right)}{\left(B_{21} A_{1}-B_{12} A_{2}\right)^{2}+A_{1} A_{2}\left(B_{11}+B_{22}\right)^{2}} \\
& \cdot\left(\begin{array}{cc}
B_{21}^{2} A_{1}+\left[B_{11} B_{22}-B_{12} B_{21}+B_{11}^{2}\right] A_{2} & B_{22} B_{21} A_{1}+B_{11} B_{12} A_{2} \\
B_{22} B_{21} A_{1}+B_{11} B_{12} A_{2} & B_{12}^{2} A_{2}+\left[B_{11} B_{22}-B_{12} B_{21}+B_{22}^{2}\right] A_{1}
\end{array}\right) .
\end{aligned}
$$


The initial values of $p_{1}, p_{2}, Q$ are determined by (5.6), (5.5), (5.9). Note that (5.9) also determines the determinant

$$
\mathrm{He}\left(x^{e}\right)=P_{11} P_{22}-P_{12} P_{21}
$$

in (4.8). Next we consider the transport equation (3.38). With (5.2a) and

$$
\sum_{i=1}^{2} \frac{\partial}{\partial x_{i}}\left(\frac{d x_{i}}{d s}\right)=\frac{d}{d s} \ln |J|,
$$

(see [8]), in which $J$ is the Jacobian

$$
J=\left|\begin{array}{ll}
\frac{d x_{1}}{d s} & \frac{\partial x_{1}}{\partial \theta} \\
\frac{d x_{2}}{d s} & \frac{\partial x_{2}}{\partial \theta}
\end{array}\right|,
$$

equation (3.38) is rewritten as

$$
\frac{d}{d s}\left(\ln w^{2}|J|\right)=-\sum_{i=1}^{2}\left[\frac{\partial b_{i}}{\partial x_{i}}+p_{i} \frac{\partial a_{i}}{\partial x_{i}}\right] .
$$

Differentiation of $(5.2 \mathrm{a}),(5.2 \mathrm{~b})$ with respect to $\theta$ leads to the equations

$$
\begin{aligned}
& \frac{d}{d s}\left(\frac{\partial x_{i}}{\partial \theta}\right)=\frac{\partial b_{i}}{\partial \theta}+\frac{\partial a_{i}}{\partial \theta} p_{i}+a_{i} \frac{\partial p_{i}}{\partial \theta}, \\
& \frac{d}{d s}\left(\frac{\partial p_{i}}{\partial \theta}\right)=-\sum_{j=1}^{2}\left[\frac{\partial^{2} b_{j}}{\partial x_{i} \partial \theta} p_{j}+\frac{\partial b_{j}}{\partial x_{i}} \frac{\partial p_{j}}{\partial \theta}+\frac{1}{2} \frac{\partial^{2} a_{j}}{\partial x_{i} \partial \theta} p_{j}^{2}+\frac{\partial a_{j}}{\partial x_{i}} \frac{\partial a_{j}}{\partial \theta} p_{j}\right],
\end{aligned}
$$

$i=1,2$, which describe the rate of change with $s$ of $\partial x_{i} / \partial \theta$ and, using (5.2a), of $J$. The initial value at $s=0$ of $w$ is chosen according to (3.36c). The initial values of $\partial x_{i} / \partial \theta$ and $\partial p_{i} / \partial \theta$ are obtained by differentiation of the initial expressions (5.4) for $x_{i}$ and (5.6) for $p_{i}$ with respect to $\theta$.

To obtain $Q_{01}, w_{01}$, the system of 10 ordinary differential equations (5.2), (5.12), (5.13) is integrated. By trial and error the angle $\theta$ of the initial point is manipulated to obtain a characteristic containing points close to $\left(0,-b_{20} / b_{22}\right)$. Once a $\left(\Delta^{2}, \Delta\right)$ neighbourhood of $\left(0,-b_{20} / b_{22}\right)$ is reached, $0<\Delta \ll 1$, the integration is terminated. Using the values of $Q, w$ obtained numerically at points near the end of the characteristic and the formulas (3.42), (3.43) for $Q$ and (3.55) for $w$, valid in the case $x_{1}=O\left(\hat{x}_{2}^{2}\right)$, $\hat{x}_{2}$ small, we approximate the values of $Q_{01}, w_{01}$.

The solutions of $Q$ and $w$ obtained by the numerical method described above are not always unique functions of $x$. By assumption, the solution is unique along the characteristic that starts at the initial point with $r=0$ and ends at $\left(0,-b_{20} / b_{22}\right)$. In numerical computations, this characteristic cannot be followed exactly. Near $\left(0,-b_{20} / b_{22}\right)$ the characteristics curve upward or downward along $x_{1}=0$ and get into caustic surfaces, as indicated by a change of sign in the determinant (5.11b). There, the solution is not a unique function of $x$. The numerical integration has been terminated before the determinant vanishes. The boundary $x_{1}=0$ cannot be approached too closely. Consequently, $\Delta$ cannot be taken arbitrarily small, which limits the accuracy of the computed values of $Q_{01}, w_{01}$. In the subsequent example, the numerical computation was stopped at $x_{1} \approx 0.02$. 
6. An example. Consider the predator-prey system defined by the diffusion

$$
\begin{aligned}
& a_{1}(x)=x_{1}\left(1.28+0.80 x_{1}+0.32 x_{2}\right), \\
& a_{2}(x)=x_{2}\left(1.08+0.28 x_{1}+0.40 x_{2}\right),
\end{aligned}
$$

and the drift

$$
\begin{aligned}
& b_{1}(x)=x_{1}\left(0.72-0.40 x_{1}-0.32 x_{2}\right), \\
& b_{2}(x)=x_{2}\left(0.12+0.28 x_{1}-0.40 x_{2}\right),
\end{aligned}
$$

in which $x_{1}$ and $x_{2}$ denote the prey and predator density, respectively. The stochastic system defined by (6.1) has previously been studied in [12]. Some trajectories of the deterministic system are depicted in Fig. 2. The numerical computation described in $\$ 5$ produces the values

$$
Q_{01}=0.26, \quad w_{01}=1.1, \quad Q_{02}=0.30, \quad w_{02}=1.5 \text {. }
$$

The projection on the $x$-plane of the characteristics (called rays), used in this computation, are depicted in Fig. 3. Outside the region $D$, the probability of exit at the boundary $x_{i}=0$ is given by $u_{i}$ :

$$
\begin{aligned}
u_{1}(x) & =\frac{0.69 e^{-0.26 / \varepsilon}+1.03 e^{-\left(0.30+x_{1} \gamma\left(x_{2}\right)\right) / \varepsilon}}{0.69 e^{-0.26 / \varepsilon}+1.03 e^{-0.30 / \varepsilon}}\left[1-e^{-\tilde{\gamma}\left(x_{1}\right) x_{2} / \varepsilon}\right] \\
& \sim 1-e^{-\tilde{\gamma}\left(x_{1}\right) x_{2} / \varepsilon}, \\
u_{2}(x) & =\frac{0.69 e^{-\left(0.26+x_{2} \tilde{\gamma}\left(x_{1}\right)\right) / \varepsilon}+1.03 e^{-0.30 / \varepsilon}}{0.69 e^{-0.26 / \varepsilon}+1.03 e^{-0.30 / \varepsilon}}\left[1-e^{-\gamma\left(x_{2}\right) x_{1} / \varepsilon}\right], \\
& \sim e^{-\tilde{\gamma}\left(x_{1}\right) x_{2} / \varepsilon},
\end{aligned}
$$

according to (3.69), (3.71). We conclude that if the starting point of the system (6.1) is outside an $O(\varepsilon)$-neighbourhood of the $x_{1}$-axis, i.e., if the initial predator density is not very small, then the prey will become extinct before the predator, with probability

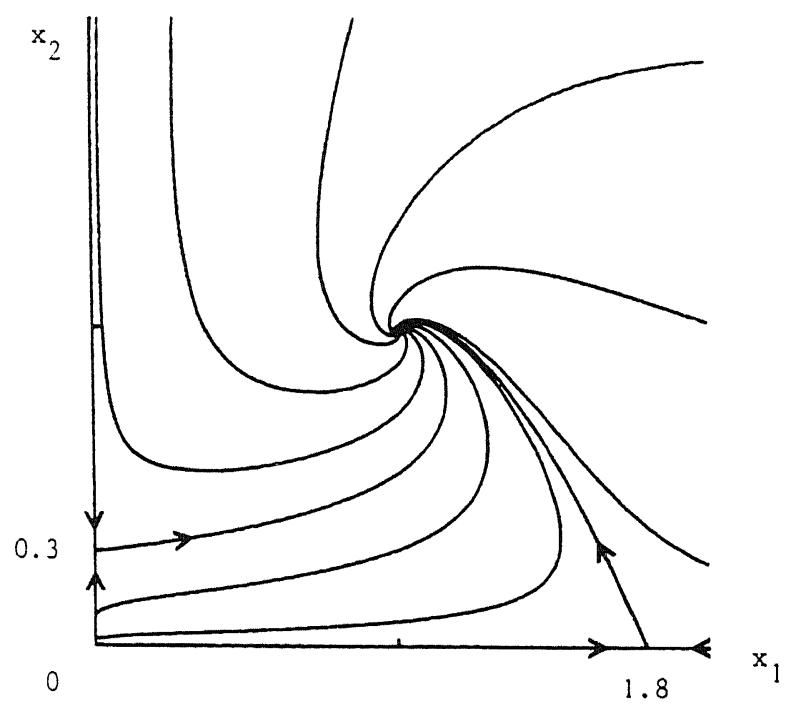

FIG. 2. Trajectories of the deterministic system associated with the stochastic system (6.1). The critical points are $(0,0),(0,0.3),(1.8,0),(1,1)$. 


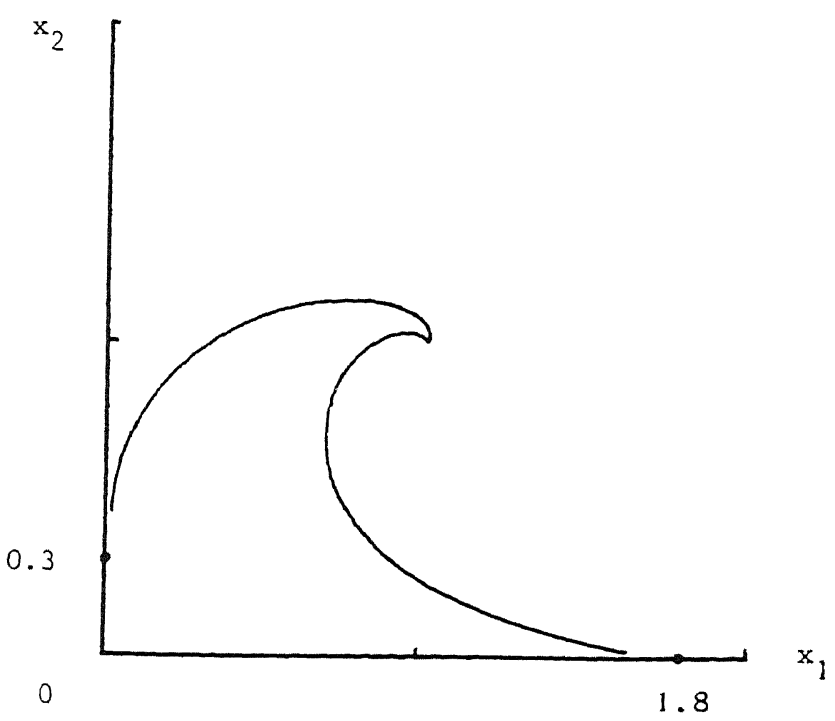

FIG. 3. The rays used in the numerical computation of $Q_{01}, w_{01}, Q_{02}, w_{02}$.

- one in the limit for $\varepsilon \rightarrow 0$. The expectation and variance of the exit time satisfy equations (4.10), uniformly in $R$, with

$$
C_{T, 1}(\varepsilon)=\frac{\sqrt{\varepsilon}}{0.11 e^{-0.26 / \varepsilon}+0.16 e^{-0.30 / \varepsilon}} \sim 9.1 \sqrt{\varepsilon} e^{0.26 / \varepsilon},
$$

which indicates that if the starting point of the system $(6.1)$ is outside $O(\varepsilon)$-neighbourhoods of the coordinate axes, i.e., if the initial prey and predator densities are not very small, the expected time of extinction of one of the populations is exponentially large. The functions $\gamma$ and $\tilde{\gamma}$ are computed numerically. The expression (3.31a) supplies the

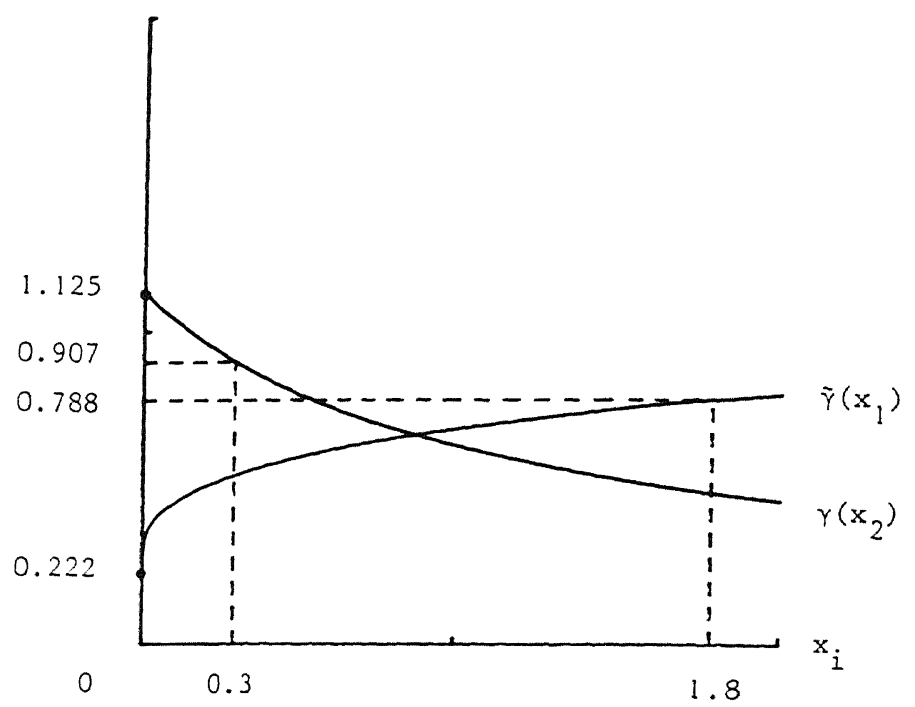

FIG. 4. The functions $\gamma, \tilde{\gamma}$. The critical points, at which the initial condition for the Bernoulli differential equation is specified, are indicated. The values denoted along the vertical axis follow from $(3.30), \lim _{\mathrm{x}_{2} \rightarrow 0} \gamma\left(x_{2}\right)=$ $2 b_{10} / a_{10}$ (see (3.31b)), and similar formulas for the other boundary. 
starting values of $\gamma$ at $-b_{20} / b_{22} \pm \eta$, where $\eta$ is a small number, and a forward (backward) finite difference scheme, based on the Bernoulli equation (3.24), (3.25) is used to obtain $\gamma\left(x_{2}\right)$ for $x_{2}>-b_{20} / b_{22}+\eta\left(x_{2}<-b_{20} / b_{22}-\eta\right)$. The graphs of $\gamma, \tilde{\gamma}$ are shown in Fig. 4. In the boundary layers along $x_{1}=0$ and $x_{2}=0$ a small (large) value of $\gamma, \tilde{\gamma}$ respectively, may be interpreted as a relatively weak (strong) stochastic stability. From Fig. 4 we conclude that for low prey density the stochastic stability of the system (6.1) decreases with increasing predator density; for low predator density the stochastic stability increases with increasing prey density.

Acknowledgments. I am grateful to Johan Grasman for many discussions on the subject treated in this paper. Thanks to both him and Huib de Swart for comments on the manuscript.

\section{REFERENCES}

[1] C. M. Bender and S. A. Orszag, Advanced Mathematical Methods for Scientists and Engineers, McGraw-Hill, New York, 1978.

[2] R. Courant And D. Hilbert, Methods of Mathematical Physics, Vol. 2, Partial Differential Equations, Interscience, New York, 1966.

[3] A. Erdélyi, W. Magnus, F. Oberhettinger, and F. G. Tricomi, Higher Transcendental Functions, Vol. 2, McGraw-Hill, New York, 1953.

[4] C. W. Gardiner, Handbook of Stochastic Methods for Physics, Chemistry and the Natural Sciences, Springer-Verlag, Berlin, New York, 1983.

[5] J. H. GILlESPIE, The interaction of genetic drift and mutation with selection in a fluctuating environment, Theoret. Pop. Biol., 27 (1985), pp. 222-237.

[6] I. S. Gradshteyn AND I. M. Ryzhik, Table of Integrals, Series, and Products, Corrected and Enlarged Edition, Academic Press, New York, 1980.

[7] F. B. HANSON AND C. TIER, An asymptotic solution of the first passage problem for singular diffusion in population biology, SIAM J. Appl. Math., 40 (1981), pp. 113-132.

[8] D. LuDwiG, Persistence of dynamical systems under random perturbations, SIAM Rev., 17 (1975), pp. 605-640.

[9] B. J. MATKOWSKY AND Z. SCHUSS, The exit problem for randomly perturbed dynamical systems, SIAM J. Appl. Math., 33 (1977), pp. 365-382.

[10] - Diffusion across characteristic boundaries, SIAM J. Appl. Math., 42 (1982), pp. 822-834.

[11] B. J. MATKOWSKY, Z. SChUSS, AND C. TIER, Diffusion across characteristic boundaries with critical points, SIAM J. Appl. Math., 43 (1983), pp. 673-695.

[12] H. ROOZEN, Equilibrium and extinction in stochastic population dynamics, Bull. Math. Biol., 49 (1987), pp. 671-696.

[13] Z. Schuss, Theory and Applications of Stochastic Differential Equations, John Wiley, New York, 1980.

[14] A. M. WAZWAZ AND F. B. HANSON, Matched uniform approximations for a singular boundary point and an interior turning point, SIAM J. Appl. Math., 46 (1986), pp. 943-961.

[15] - Singular boundary resonance with turning point resonance, SIAM J. Appl. Math., 46 (1986), pp. 962-977. 